\title{
Cockayne Syndrome with ERCC8 Gene Mutation: A Case Report
}

\author{
KANIJ FATEMA ${ }^{1}$, MD MIZANUR RAHMAN ${ }^{2}$, SHAHEEN AKHTER ${ }^{3}$
}

\begin{abstract}
:
Cockayne syndrome (CS) is a genetic disorder characterized by growth failure, microcephaly, cognitive delay, visual and hearing disorders. Patients usually present with dysmorphism and global delay. It is an autosomal recessive disorder, mutation of two genes ERCC8 and ERCC6 were observed. We report a 4 year old child who was diagnosed as a case of Cockayne syndrome, based on clinical, neuroimaging and genetic study findings. This case had growth failure, dysmorphism, optic atrophy, global developmental delay, intracerebral calcification and mutation of ERCC8 gene.
\end{abstract}

Key words: Cockayne syndrome, ERCC8 mutation, growth failure, dysmorphism

\section{Introduction:}

Cockayne syndrome (CS) is a rare genetic disorder involving predominantly nervous system, skin,growth and eyes. It was first described by Cockayne in 1936. ${ }^{1,2}$ Very limited cases have been reported till that worldwide. The key features of this degenerative disorder are microcephaly, growth failure, hearing loss, cataract, retinal dystrophy and developmental delay. Other important features are skin photosensitivity, dental caries, wizened facial appearance and intracerebral calcification. . $^{3,4}$ Some patients may have thyroid hormone deficiency, hypertension and renal failure. Progression of CS leads to functional debility and bed ridden in the second decade of life. The average life expectancy is 12.5 year; common cause of death is infection. ${ }^{5}$

This disorder is associated with a specific defect in the ability to perform transcription-coupled repair of active genes after UV irradiation. There are three main subdivisions of Cockayne syndrome, Classical form Type-A, Congenital form Type-B, Late onset form Type$C$, A fourth form now recognized as Xeroderma pigmentosa-Cockayne syndrome (XP-CS). The CS-A is caused by homozygous or compound heterozygous mutation in the gene encoding the group 8 excision-

1. Associate Professor, Department of Pediatric Neurology, IPNA, BSMMU, Dhaka

2. Ex-Chairman and Professor, Department of Pediatric Neurology, BSMMU, Dhaka

3. Professor, Department of Pediatric Neurology, Director, IPNA, BSMMU, Dhaka

Correspondence: Dr. Kanij Fatema, Associate Professor, Department of Pediatric Neurology, Institute of Pediatric Neurodisorder and Autism (IPNA), Bangabandhu Sheikh Mujib Medical University, Dhaka, Bangladesh. Cell: +8801713097751 email-maiomonami@gmail.com

Received: 29/08/2020 repair cross-complementing protein (ERCC8; 609412) on chromosome $5 q 11$, and the CS- B (133540) is caused by mutation in the ERCC6 gene (609413) on chromosome 10q11.5,6 Early diagnosis is important for genetic counseling. Here we present a case of CS-A with genetic profile.

\section{Case report:}

A 4 year old boy presented to a tertiary care referral hospital for evaluation. The boy had a history of delay in development and growth failure. He was the only issue of nonconsanguineous parents. He was born by lower uterine caesarian section, term, birth weight was $2.6 \mathrm{~kg}$. He cried immediately after birth. There was no history of antenatal and perinatal insult. None of his family members had similar type of illness. He had no history of seizure or movement disorder. In developmental history, he developed neck control at 8 months, started to sit at 18 month of age, could stand with support at 3 year of age. He did not learn to walk yet. He could speak only 3-5 meaningful words. His hearing was intact but his vision was impaired.

On examination, he had microcephaly, dysmorphic facies, thin and sparse hair, sunken eyes, prominent nose and ears, wizened look, no neuro-cutaneous mark. He was anthropometrically mildly wasted and moderately stunted. He was vitally stable. Eye examination revealed: anterior segment was enopthalmic, in posterior segment, there was pigmentary change in retina and optic atrophy. Hearing and dental examination was normal. Neurological examination revealed that, bulk of muscle was decreased, tone was increased and power $4 / 5$ in both upper and lower limbs. Gait could not be evaluated as he could not walk. Other systemic 


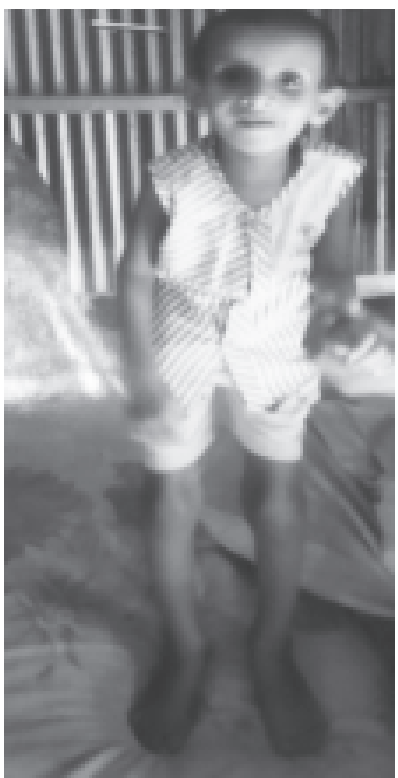

Fig.-1: Dysmporphic child with short stature and microcephaly

examination revealed normal. His psychological assessment was done by WISC-R IV which revealed moderate intellectual disability. While assessing his activity of daily living, it was found that, he could not eat, bath on his own, he was toilet trained.

CT scan of brain showed there was bilateral calcification of lentiform nucleus, head of caudate nucleus. There was also curvilinear calcifications at the sulcal depths involving the frontal, parietal and occipital lobes and bilateral paucity of white matter. (Figure 2) There was generalized cortical atrophy. EEG was normal. Basic metabolic test, serum uric acid, liver function test, renal function test, serum

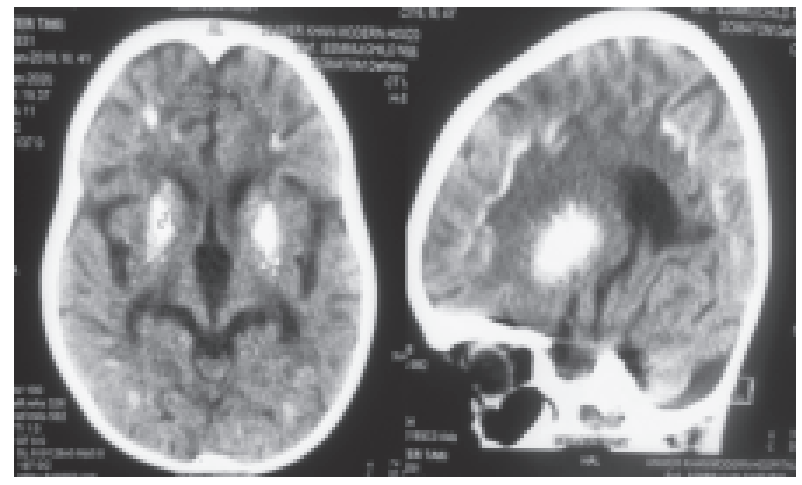

(A)

(B)

Fig.-2 : A. CT scan of brain, axial section showing bilateral lentiform nucleus calcification. B. sagital section showing calcification of thalamus, culvilinear calcification at sulcal depth of frontal, parietal and occipital areas. electrolytes, serum calcium, phosphate level, thyroid hormone level, random blood sugar were normal.

Clinical exome sequencing revealed: mutation of ERCC8 [Exon 2, c. (1705+1_1706-1)_(1845+1_18461) del (Exonic deletion), homozygous] suggestive of Cockayne syndrome A. We treated the patient with supportive management. Patient was enrolled for speech and language therapy, occupational therapy, visual stimulation, physiotherapy and special schooling. The parents were counseled regarding the nature of the disease, chance of recurrence and were suggested for genetic tests for the parents.

\section{Discussion}

There are very limited cases of CS with genetic diagnosis. This rare syndrome has involvement of multiple systems. Our patient had neurological involvement predominantly along with dysmorphism. The features in other reported cases were sensorineural hearing loss, hepatic dysfunction, cholestasis, poor peripheral circulation leading to cold periphery and recurrent respiratory tract infection. ${ }^{7-11}$ None of these features were observed in our patient. However, our patient had the two cardinal features of CS: microcephaly and growth failure.Postnatal growth failure is an important clinical feature of CS. Wilson BT et al found significant patients had growth failure; the growth parameters were; height $-6.62 \pm 3.09 \mathrm{SD}$; weight $-6.62 \pm 4.79$ SD. Microcephaly is another predominant feature of $\mathrm{CS}$ which have been observed in several studies. The microcephaly in CS is progressive which is well documented at 1 to 2 years of age. ${ }^{12}$

Opthalmological findings are another important parameter in CS. The abnormalities are cataract, photophobia, retinal dystrophy, pigmentary retinopathy, enophthalmos, strabismus, amblyopia, optic atrophy, nystagmus, corneal lesions, band keratopathy, recurrent erosions, and poor pupillary response to dilating agents. Our patient had optic atrophy along with pigmentary change in the retina. 4,13

Previous study has shown that developmental delay contributed to musculoskeletal deformity like contracture which was absent in this cases. ${ }^{12}$ Another frequently observed feature was feeding difficulty contributing to growth failure. This feeding difficulty is mainly due to gastroesopahgeal reflux disease. Other less common feature was impaired glucose tolerance test, photosensitive rash and dental caries. ${ }^{6,12}$ 
Neurodevelopmental impairment was the key feature for which our patient consulted. He had significant delay in most of the domains of development namely speech, motor, cognition and ADL. In most of the reported cases there was developemtal delay. $2,6,12$ Moreover in some reported cases there were seizure, tremor and neuroregression which were absent in our case. ${ }^{6,12}$

Regarding neuroimaging, our patient had a significant finding of calcification in different areas of brain. About $85 \%$ patients of study done by Wilson BT et al found abnormal neuroimaging, calcification was present in $55 \%$. The pattern of calcification was unique here involving basal ganglia, calvilinear pattern of involvement of the subcortical white matter which has also been observed in our case. Other features found in neuroimaging are white matter demyelination, hypomyelination, cerebellar hypoplasia or atrophy, corpus callosal thinning etc. $6,12,14$

The two genes associated with CS are CKN1 or ERCC8 and ERCC6 respectively. These genes are also associated with mutations in XPB (Xeroderma pigmentosum $\mathrm{B}$ ) gene, XPD (Xeroderma pigmentosum D) gene and XPG (Xeroderma pigmentosum $G$ ) gene. Our case was associated with mutation of ERCC8 mutation. The other disorders associated with the genetic mutation should be keep in mind and differentiated. ${ }^{15-18}$

The management of CS is mainly supportive as currently there is no cure. Periodic surveillance is important to maximize the quality of life. Growth monitoring, nutritional supplementation, cognitive behavioural therapy, special educations are mainstay of treatment along with genetic counseling. 5,12

\section{Conclusion:}

CS is a rare genetic disorder of childhood. It is suggested that CS should be suspected if there is dysmorphism, microcephaly, and growth failure, visual and hearing impairment. Genetic study should be done to confirm and for counseling.

\section{References:}

1. Cockayne EA. Dwarfism with retinal atrophy and deafness. Arch Dis Child 1936; 11:1-8.

2. Ozdirim E, Topcu M, Ozon A, Cila A. Cockayne syndrome: Review of 25 cases. PediatrNeurol1996;15:312-6.
3. Kleijer WJ, Laugel V, Berneburg M, Nardo T, Fawcett $H$, Gratchev A, et al. Incidence of DNA repair deficiency disorders in western Europe: Xerodermapigmentosum, Cockayne syndrome and trichothiodystrophy. DNA Repair (Amst) 2008;7:744-50.

4. Nance MA, Berry SA. Cockayne syndrome: Review of 140 cases. Am J Med Genet 1992; 42:68-84.

5. BaratalM ,Souza RO, Tavares FLS, Gonçalves CS , Garcia $\mathrm{LL}$, Almeida CP et al. Cockayne Syndrome -a case report. Brazilian Journal of Medicine and Human Health 2014 ;2:50-2.

6. Maiya AS, Shenoy S, Jayaram R, Madan R, Kumar V. Cockayne Syndrome: A Rare Case Report. Int J Med Health Sci. 2014,3;92-4.

7. Laugel V. Cockayne syndrome: the expanding clinical and mutational spectrum. Mech Ageing Dev 2013;134:16170.

8. Abdel Ghaffar TY, Elsobky ES, Elsayed SM. Cholestasis in patients with Cockayne syndrome and suggested modified criteria for clinical diagnosis. Orphanet J Rare Dis $2011 ; 6: 13$.

9. Andrade LN, Nathanson JL, Yeo GW, Menck CF, Muotri AR. Evidence for premature aging due to oxidative stress in iPSCs from Cockayne syndrome. Hum Mol Genet 2012;21:3825-34.

10. Pasquier L, Laugel V, Lazaro L, Dollfus H, Journel H, Edery $P$, et al. Wide clinical variability among 13 new Cockayne syndrome cases confirmed by biochemical assays. Arch Dis Child. 2006 ;91:178-82.

11. Ovaert C, Cano A, Chabrol B. Aortic dilatation in Cockayne syndrome. Am J Med Genet A 2007;143:2604-6.

12. Wilson BT, Stark Z, Sutton RE, Danda S, Ekbote AV, et al. The Cockayne Syndrome Natural History (CoSyNH) study: clinical findings in 102 individuals and recommendations for care. Genet Med. 2016 ;18:483-93.

13. Traboulsi El, DeBecker I, Maumenee IH. Ocular findings in Cockayne Syndrome. Am J Ophthalmol.1992;114:579-83.

14. Weidenheim KM, Dickson DW, Rapin I. Neuropathology of Cockayne syndrome: evidence for impaired development, premature aging, and neurodegeneration. Mech Ageing Dev 2009;130:619-36.

15. Lehmann AR. DNA repair-deficient diseases, xerodermapigmentosum, Cockayne syndrome and trichothiodystrophy. Biochimie 2003;85:110-1.

16. Gorlin RJ, Cohen MM, Hennekam RCM. Syndromes of the Head and Neck. New York: Oxford University Press; 2001. p. $596-600$.

17. Shiomi N, Kito S, Oyama M, Matsunaga T, Harada YN, Ikawa $\mathrm{M}$, et al. Identification of the XPG region that causes the onset of Cockayne syndrome by using Xpg mutant mice generated by the cDNA-mediated knock-in method. Mol Cell Biol 2004; 24:3712-9.

18. Tuo J, Chen C, Zeng X, Christiansen M, Bohr VA. Functional crosstalk between hOgg1 and the helicase domain of Cockayne syndrome group B protein. DNA Repair (Amst). 2002;1:913-27. 